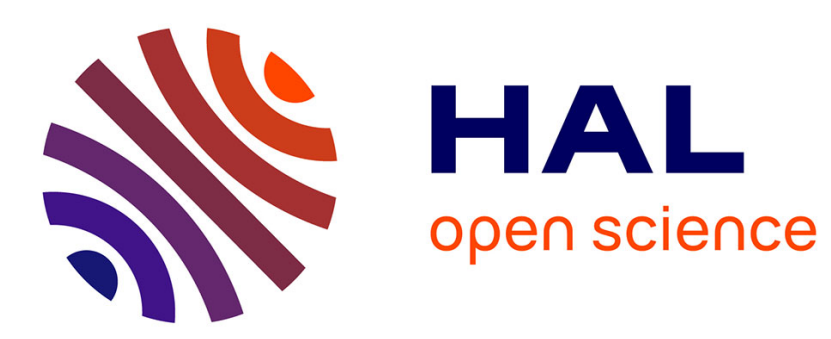

\title{
A Parallel time-domain Maxwell solver using upwind schemes and triangular meshes
}

Jean-Pierre Cioni, Loula Fatima Fezoui, Hervé Steve

\section{To cite this version:}

Jean-Pierre Cioni, Loula Fatima Fezoui, Hervé Steve. A Parallel time-domain Maxwell solver using upwind schemes and triangular meshes. [Research Report] RR-1867, INRIA. 1993. inria-00074806

\section{HAL Id: inria-00074806 \\ https://hal.inria.fr/inria-00074806}

Submitted on 24 May 2006

HAL is a multi-disciplinary open access archive for the deposit and dissemination of scientific research documents, whether they are published or not. The documents may come from teaching and research institutions in France or abroad, or from public or private research centers.
L'archive ouverte pluridisciplinaire HAL, est destinée au dépôt et à la diffusion de documents scientifiques de niveau recherche, publiés ou non, émanant des établissements d'enseignement et de recherche français ou étrangers, des laboratoires publics ou privés. 


\title{
A parallel time-domain maxwell solver using upwind schemes and \\ triangular meshes
}

\author{
Jean-Pierre CIONI \\ Loula FEZOUI \\ Hervé STEVE

$$
\begin{aligned}
& N^{\circ} \mathbf{1 8 6 7} \\
& \text { Mars } 1993
\end{aligned}
$$

PROGRAMME 6

Calcul Scientifique, Modélisation et

Logiciels numériques

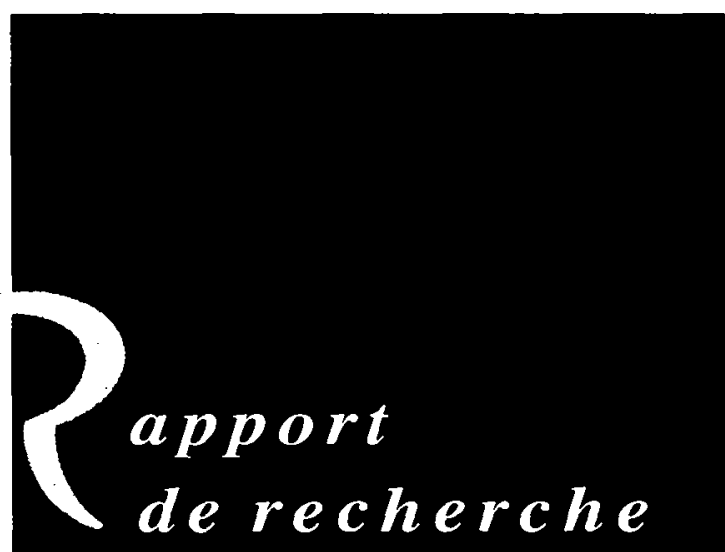




\title{
A PARALLEL TIME-DOMAIN MAXWELL SOLVER USING UPWIND SCHEMES AND TRIANGULAR MESHES
}

\author{
J.P. CIONI ${ }^{*}$ - L. FEZOUI ** - H. STEVE ${ }^{* * *}$
}

* INRIA Sophia-Antipolis

2004, Route des Lucioles B.P. 93

06902 Sophia-Antipolis Cedex, France

** CERMICS-INRIA Sophia-Antipolis

2004, Route des Lucioles B.P. 93

06902 Sophia-Antipolis Cedex, France

*** DASSAULT AVIATION

78, quai M.Dassault

92214 Saint-Cloud, France 


\title{
A Parallel Time-Domain Maxwell Solver Using Upwind Schemes And Triangular Meshes
}

\author{
Jean-Pierre CIONI, Loula FEZOUI, Hervé STEVE
}

\begin{abstract}
:
A Finite-Volume/Finite-Element method developed in CFD is applied to solve numerically the time-dependent 2D Maxwell system. We use a third-order accurate explicit scheme with triangular meshes.

We are particularly concerned here with scattering problems for both homogeneous and non-homogeneous materials. Radar Cross Section calculations are presented and compared with exact or integral method solutions. A parallel version of the algorithm on the Connection Machine is presented and the performances are compared with those obtained with a fully vectorized version on the CRAY YMP.
\end{abstract}

\section{Un solveur parallel de Maxwell instationnaire utilisant des schémas décentrés en maillages triangulaires}

\section{Résumé:}

Une approximation mixte Eléments-finis/Volumes-finis développée en mécanique des fluides est employée ici pour la résolution numérique des équations de Maxwell instationnaires en dimension deux d'espace. Nous utilisons un schéma explicite précis à l'ordre trois avec des maillages triangulaires.

Nous traîtons ici des problèmes de diffraction d'onde aussi bien en milieu homogène que hétérogène. Des calculs de Surface Equivalente Radar sont présentés et comparés aux solutions exactes ou bien aux solutions numériques d'une méthode intégrale. Une version parallèlle de l'algorithme sur la Connection Machine est présentée et les performances sont comparées à celles obtenues sur le CRAY YMP avec une version entièrement vectorisée. 


\section{Contents}

Introduction 1

1 Maxwell equations 2

1.1 Conservative form and hyperbolicity ............. 2

1.2 Boundary conditions ................... 4

2 Numerical approximation $\mathbf{5}$

2.1 Variational formulation . . . . . . . . . . . . . 5

2.2 Steger-Warming flux decomposition . . . . . . . . . . 6

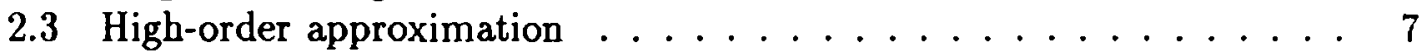

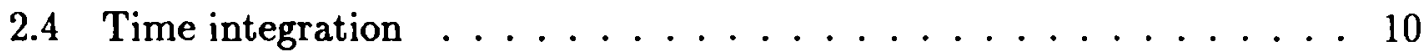

2.5 Radar Cross Section evaluation ................ 10

2.6 Convergence to the steady state . . . . . . . . . . . . 11

2.7 Divergence error . . . . . . . . . . . . . . . . 13

3 Numerical experiments 14

3.1 Unstationary experiments ................... 14

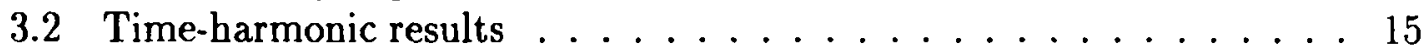

4 Parallel implementation on the Connection Machine 20

4.1 Presentation of the $\mathrm{CM} 2 / 200 \ldots \ldots \ldots 20 . \ldots . \ldots 2 \ldots$

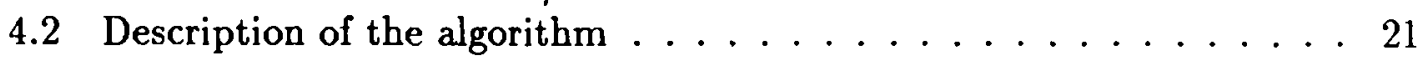

4.3 Performance results and comparisons . . . . . . . . . . 21

5 Conclusion $\quad 24$

$\begin{array}{ll}\text { References } & \mathbf{2 5}\end{array}$ 


\section{Introduction}

The interest of the Computational Electromagnetics (CEM) community in timedomain simulations is increasing nowadays.

At the same time we witness a transfer of the most recent and sophisticated methods developed in the Computational Fluid Dynamics (CFD) into the CEM domain [23]. The main justification of such a transfer lies in the hyperbolicity of the time-domain Maxwell system which may be expressed as a set of conservation laws when written in total field form. The use of the scattered field variables will just introduce incident wave terms in the right-hand-side of the system and thus will not cause any difficulty when applying a numerical method constructed for solving the Euler system $[9,12,13]$.

However some questions have to be discussed such as the divergence-free conditions which are not usually considered for an Euler solver and also the ability of such a solver to capture wcll the Maxwell solutions in the frequency-domain. In the latter case, one has also to find some good criterion of convergence for the time-domain solver. These questions will be discussed in this paper and some solutions will be proposed.

The time-domain solver presented here is based on a mixed Finite-Volume/FiniteElement method using a third-order accurate upwind scheme. The time integration is done using a three stage explicit Runge-Kutta scheme. These ingredients result in a high-order accurate scheme both in time and space $[6,7]$.

The method is successfully used to compute numerical solutions of 2-D Maxwell system in both frequency- and time-domain with homogeneous or non-homogeneous materials. The computed solutions are compared with the analytical ones when possible or with the corresponding integral method solutions otherwise. We have also used our previous works in parallelising the same algorithm applied to Euler and Navier-Stokes simulations [24] to present here parallel computations in electromagnetics on a massively parallel machine: the Connection Machine CM2/200. Performance results in CPU time and Mflops (millions of operations per second) of the parallel solver are compared to those obtained with a fully vectorized version on the CRAY-YMP. 


\section{Maxwell equations}

\subsection{Conservative form and hyperbolicity}

Electromagnetic scattering problems are governed by Maxwell equations, which can be written in vector form as:

$$
\left\{\begin{array}{l}
\frac{\partial \mathbf{B}(\mathbf{x}, t)}{\partial t}+\operatorname{rot}(\mathbf{E}(\mathbf{x}, t))=0 \\
\frac{\partial \mathbf{D}(\mathbf{x}, t)}{\partial t}-\operatorname{rot}(\mathbf{H}(\mathbf{x}, t))=-\mathbf{j}(\mathbf{x}, t)
\end{array}\right.
$$

where $\mathbf{B}$ is the magnetic field induction, $\mathbf{D}$ the electric field displacement, $\mathbf{E}$ and $\mathbf{H}$ the electric and magnetic field intensities respectively, and $\mathbf{j}$ the current density. $\mathbf{D}$ and $\mathbf{B}$ also satisfy the divergence conditions:

$$
\operatorname{div}(\mathbf{D})=\rho \quad \text { and } \operatorname{div}(\mathbf{B})=0
$$

The electric charge $\rho$ satisfies the equation:

$$
\frac{\partial \rho}{\partial t}+\operatorname{div}(\mathbf{j})=0
$$

We recall that the divergence conditions are redundant with equations (1) by considering the conservation law of electric charge (3) and an initial value satisfying Eq. (2) (see [8] for example). Hence, we only take into account equations (1), assuming here no current and charge densities $(\rho=0, \mathbf{j}=0)$.

$\mathbf{D}$ and $\mathbf{B}$ are related to $\mathbf{E}$ and $\mathbf{H}$ through the permittivity $\varepsilon(\mathbf{x})$ and the permeability $\mu(\mathbf{x})$ as:

$$
\mathbf{D}=\varepsilon(\mathbf{x}) \mathbf{E} \quad \text { and } \quad \mathbf{B}=\mu(\mathbf{x}) \mathbf{H}
$$

which corresponds to linear isotropic materials.

In two dimensions, Maxwell equations can be split into two sets of systems associated to transverse magnetic $(T M)$ and tranverse electric $(T E)$ waves. In this paper, we deal only with two-dimensional scattering cases. However, the method to be presented can be extended to the most general three-dimensional problems.

As equations are linear, we will now consider the formulation in scattered fields with an incident wave solution of Maxwell equations in free space. In this paper, we will consider the conservative form of Maxwell equations which implies recasting the system in function of the fields $\mathbf{D}$ and $\mathbf{B}$. Hence, the conservative scheme (described in section 2) will be employed for solving efficiently field discontinuities in case of non-homogeneous media. One may easily check that the non-conservative form involving $(\mathbf{E}, \mathbf{H})$ fields and the conservative one are equivalent in case of homogeneous media.

In the $T M_{z}$ case $(\mathbf{H . z}=0)$, one has $\mathbf{B}=\left(B_{x}, B_{y}, 0\right)$ and $\mathbf{D}=\left(0,0, D_{z}\right)$ in cartesian 
coordinates. Only the non-zero components of the electric and magnetic inductions are considered. Equations (1) and (4) then lead via an appropriate change of variables to the following conservative form written in scattered field:

$$
\mathbf{Q}_{\tau}+\mathbf{F}(\mathbf{Q})_{x}+\mathbf{G}(\mathbf{Q})_{y}=\mathbf{S}^{\text {inc }}
$$

with:

$$
\begin{gathered}
\mathbf{Q}=\left\{\begin{array}{l}
Q_{1} \\
Q_{2} \\
Q_{3}
\end{array}\right\}=\left\{\begin{array}{c}
c_{0} B_{x} \\
c_{0} B_{y} \\
D_{z} / \varepsilon_{0}
\end{array}\right\} \\
\mathbf{F}(\mathbf{Q})=\left\{\begin{array}{c}
0 \\
-Q_{3} / \varepsilon_{r} \\
-Q_{2} / \mu_{r}
\end{array}\right\} ; \mathbf{G}(\mathbf{Q})=\left\{\begin{array}{c}
Q_{3} / \varepsilon_{r} \\
0 \\
Q_{1} / \mu_{r}
\end{array}\right\}
\end{gathered}
$$

and $\mathbf{S}^{i n c}=-\mathbf{Q}_{\tau}^{i n c}-\mathbf{F}\left(\mathbf{Q}^{i n c}\right)_{x}-\mathbf{G}\left(\mathbf{Q}^{i n c}\right)_{y}$ where $\mathbf{Q}^{i n c}$ denotes the incident field.

In order to deal with non-dimensional material properties, we have performed the following change of variables by introducing the permittivity $\varepsilon_{0}$ and the permeability $\mu_{0}$ of the free space:

$$
\varepsilon(\mathbf{x})=\varepsilon_{0} \varepsilon_{r}(\mathbf{x}) \text { and } \mu(\mathbf{x})=\mu_{0} \mu_{r}(\mathbf{x})
$$

The relative speed of light is $c_{r}=\frac{1}{\sqrt{\varepsilon_{r} \mu_{r}}}$. In free space, we have $\varepsilon_{r}=\mu_{r}=c_{r}=1$ and the light speed is $c_{0}=\frac{1}{\sqrt{\varepsilon_{0} \mu_{0}}}$.

We note $\tau=c_{0} t$ the new time variable which is now expressed in terms of wave lengths. The right-hand-side of (5), induced by the incident wave $\mathbf{Q}^{\text {inc }}$, is equal to zero in free space according to our assumptions.

The second point to be noticed is the hyperbolicity of Maxwell system. Let us write (5) as:

$$
\mathbf{Q}_{\tau}+\vec{\nabla} \cdot \mathbf{F}(\mathbf{Q})=\mathbf{S}^{\text {inc }}
$$

with $\mathbf{F}(\mathbf{Q})=(\mathbf{F}(\mathbf{Q}) ; \mathbf{G}(\mathbf{Q}))^{t}$. The non-conservative form of the left-hand-side of $(6)$ writes as:

$$
\mathbf{Q}_{\tau}+\vec{\nabla} \mathbf{Q} \cdot \mathbf{F}^{\prime}(\mathbf{Q})=\mathbf{S}^{\text {inc }}
$$

where $\mathbf{F}^{\prime}=\left(\frac{\partial}{\partial \mathbf{Q}} \mathbf{F}(\mathbf{Q}) ; \frac{\partial}{\partial \mathbf{Q}} \mathbf{G}(\mathbf{Q})\right)^{t}$ and $\vec{\nabla} \mathbf{Q}=\left(\frac{\partial \mathbf{Q}}{\partial x} ; \frac{\partial \mathbf{Q}}{\partial y}\right)^{t}$.

We now consider the following linear combination $\mathcal{F}(\mathbf{Q}, \boldsymbol{\eta})=\boldsymbol{\eta} . \mathbf{F}(\mathbf{Q})$ where $\boldsymbol{\eta}=\left(\eta_{1}, \eta_{2}\right)$ is any non-zero constant vector in $\mathbb{R}^{2}$. The jacobian matrix $\mathcal{A}$ defined as $\mathcal{A}(\mathbf{Q}, \boldsymbol{\eta})=$ $\boldsymbol{\eta} . \boldsymbol{F}^{\prime}(\mathbf{Q})$ is given by:

$$
\mathcal{A}=\left(\begin{array}{ccc}
0 & 0 & \eta_{2} / \varepsilon_{r} \\
0 & 0 & -\eta_{1} / \varepsilon_{r} \\
\eta_{2} / \mu_{r} & -\eta_{1} / \mu_{r} & 0
\end{array}\right)
$$


and its real eigenvalues are:

$$
\lambda_{1}=c_{r}|\boldsymbol{\eta}|, \lambda_{2}=-c_{r}|\boldsymbol{\eta}| \text { and } \lambda_{3}=0 \text {. }
$$

Concerning the $T E_{z}$ case $(\mathbf{E . z}=0)$, the non-zero components of the electromagnetic field lead to the following conservative variables:

$$
\mathbf{Q}=\left(-D_{x} / \varepsilon_{0},-D_{y} / \varepsilon_{0}, c_{0} B_{z}\right)^{t}
$$

We then obtain:

$$
\mathbf{F}(\mathbf{Q})=\left\{\begin{array}{c}
0 \\
-Q_{3} / \mu_{r} \\
-Q_{2} / \varepsilon_{r}
\end{array}\right\} \quad ; \quad \mathbf{G}(\mathbf{Q})=\left\{\begin{array}{c}
Q_{3} / \mu_{r} \\
0 \\
Q_{1} / \varepsilon_{r}
\end{array}\right\}
$$

One can easily deduce the corresponding jacobian by interchanging $\varepsilon_{\mathrm{r}}$ and $\mu_{\mathrm{r}}$ in the $T M$ case jacobian (7). The eigenvalues remain the same as in the $T M$ case and are defined by (8).

Maxwell system (5) is then of hyperbolic type and also a law-conservation system for $T M$ and $T E$ modes. These charasteristics of Maxwell system (conservativity and hyperbolicity) lead up naturally to the use of upwind schemes known to be well adapted to solve numerically similar systems in CFD (see $[1,2,5]$ for example).

\subsection{Boundary conditions}

\section{Perfectly conducting surface}

For a perfectly conducting surface, the boundary condition in terms of scattered field writes as:

$$
\boldsymbol{n} \wedge \mathbf{E}=-\boldsymbol{n} \wedge \mathbf{E}^{\text {inc }}
$$

where $\boldsymbol{n}$ is the outgoing unit normal vector of the surface.

\section{Absorbing boundary conditions}

Numerically, we have to restrict the computations to a finite domain whereas an infinite domain is required. Many authors have developed investigations to define well-posed boundary value problems. Some perfectly absorbing conditions have been set up (no parasite waves) but they cannot be used numerically for they are not local. However, a set of absorbing local boundary conditons which approximate the perfectly absorbing ones is now well known for the wave equation and Maxwell system (see [11]). We use here the first-order absorbing condition of Silver-Müller:

$$
\boldsymbol{n} \wedge \mathbf{E}=-\sqrt{\frac{\mu_{0}}{\varepsilon_{0}}} \boldsymbol{n} \wedge(\boldsymbol{n} \wedge \mathbf{H})
$$

on a boundary surface in free space. This is a practical radiation condition to implement but the outer boundary must be set far enough away so that the eventual parasite reflections do not interact with the scattering object. This is usually 
achieved by setting the boundary two wave lengths far from the object. Thus, the implementation of this boundary condition would lead to a need of a large amount of memory when dealing with three-dimensional problems. We work on adapting the second-order Joly-Mercier condition [10] to our scheme which allows an outer boundary to be approximately one wave length far from the obstacle.

\section{Numerical approximation}

A large family of approximate Riemann solvers has been developed in CFD and these schemes have proven their efliciency in solving problems where field discontinuities appear. This class of schemes seems to be well adapted to the conservative form of the time-dependent Maxwell system, particularly when dealing with heterogeneous media. We focalise our studies on high-order upwind schemes both in time and space, which allow to treat arbitrary complicated geometries and are well adapted to vector and parallel computing. We describe briefly in the following section the Finite-Volume/Finite-Element method applied to the Maxwell system. For more details on this method and its applications in CFD fields, one may refer to $[4,5]$ for example.

\subsection{Variational formulation}

Let $\mathcal{T}_{h}$ be a standard finite element triangulation of $\Omega_{h}$, the polygonal approximation of a computational domain $\Omega$. At each node $S_{\boldsymbol{i}}$, a cell $C_{\boldsymbol{i}}$ is constructed as shown in Fig. 1. The union of all the cells forms a new partition of $\Omega_{h}$ :

$$
\Omega_{h}=\bigcup_{i=1}^{n s} C_{i}
$$

The following discrete spaces are introduced:

$$
\begin{aligned}
& V_{h}=\left\{v_{h}\left|v_{h} \in C^{0}(\Omega), v_{h}\right|_{T} \in P^{1}, \forall T \in \mathcal{T}_{h}\right\} \\
& W_{h}=\left\{v_{h}\left|v_{h} \in L^{2}(\Omega), v_{h}\right|_{C_{i}}=\text { constant }, i=1, n s\right\}
\end{aligned}
$$

where $P^{1}$ is the space of piecewise linear functions.

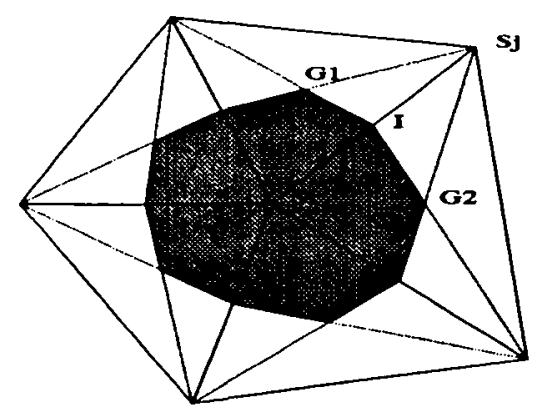

Figure 1: Cell construction in unstructured grid 
We consider the initial and boundary value problem:

$$
\begin{cases}\mathbf{Q}_{\boldsymbol{\tau}}+\vec{\nabla} . \mathbf{F}(\mathbf{Q})=\mathbf{S}^{i n c} & (\mathbf{x}, \tau) \in \Omega \times \mathbb{R}^{+} \\ \boldsymbol{n} \wedge \mathbf{E}=-\boldsymbol{n} \wedge \mathbf{E}^{i n c} & (\mathbf{x}, \tau) \in \Gamma_{b} \times \mathbb{R}^{+} \\ \boldsymbol{n} \wedge \mathbf{E}=-\sqrt{\frac{\mu_{0}}{\varepsilon_{0}}} \boldsymbol{n} \wedge(\boldsymbol{n} \wedge \mathbf{H}) & (\mathbf{x}, \tau) \in \Gamma_{\infty} \times \mathbb{R}^{+} \\ \mathbf{Q}(\mathbf{x}, \tau=0)=\mathbf{Q}_{\mathbf{0}}(\mathbf{x}) & \mathbf{x} \in \Omega\end{cases}
$$

where $\Gamma_{b}$ is a perfectly conducting surface and $\Gamma_{\infty}$ is the artificial boundary. Besides, $\mathbf{Q}_{0}$ satisfies the divergence conditions (2). A variational formulation of (11) is:

$$
\begin{gathered}
\text { Find } \mathbf{Q}_{h} \in\left(V_{h}\right)^{3}, \forall \phi_{h} \in V_{h} \\
\int_{\Omega}\left(\mathbf{Q}_{h}\right)_{\tau} \phi_{h} d x d y+\int_{\Omega} \vec{\nabla} \cdot \mathbf{F}\left(\mathbf{Q}_{h}\right) S\left(\phi_{h}\right) d x d y=\int_{\Omega} \mathbf{S}_{h}^{i n c} S\left(\phi_{h}\right) d x d y
\end{gathered}
$$

where $S$ is a bijection between spaces $V_{h}$ and $W_{h}$ defined by

$$
\forall \phi \in V_{h}, S(\phi)=\sum_{i=1, n_{s}} \phi\left(S_{i}\right) \chi\left(C_{i}\right)
$$

We recall that $\chi(\theta)$ is the charasteristic function of $\theta$ for every domain $\theta \in \Omega$.

By choosing $\phi_{h}$ as the basis $P^{1}$ function associated to the node $S_{i}$ and applying a mass-lumping to the time derivatives, we get the following system:

$$
\left.\operatorname{Area}\left(C_{i}\right)\left(\mathbf{Q}_{h}\right)_{\tau}\right|_{s_{i}}+\int_{C_{i}} \vec{\nabla} \cdot \mathbf{F}\left(\mathbf{Q}_{h}\right) d x d y=\mathbf{W}^{i n c}
$$

where $\mathbf{W}^{\text {inc }}$ denotes the incident wave contribution which will be discussed later:

$$
\mathbf{W}^{\text {inc }}=\int_{C_{\boldsymbol{i}}} \mathbf{S}_{h}^{\text {inc }} d x d y
$$

Using now a Green's formula, equation (13) leads to:

$$
\begin{array}{rlr}
\left.\operatorname{Area}\left(C_{i}\right)\left(\mathbf{Q}_{h}\right)_{\tau}\right|_{s_{i}=} & -\sum_{j \in K(i)} \int_{\partial C_{i j}} \mathbf{F}\left(\mathbf{Q}_{h}\right) \cdot \boldsymbol{\nu}_{i j} d \sigma & <1> \\
& -\int_{\partial C_{i} \cap \Gamma_{b}} \mathbf{F}\left(\mathbf{Q}_{h}\right) \cdot \boldsymbol{n} d \sigma \quad<2> \\
& -\int_{\partial C_{i} \cap \Gamma_{\infty}} \mathbf{F}\left(\mathbf{Q}_{h}\right) \cdot \boldsymbol{n} d \sigma \quad<3> \\
& +\mathbf{W}^{i n c},<4
\end{array}
$$

where $\nu_{i j}$ is the outward normal to the interface $\partial C_{i j}$ between two cells $C_{i}$ and $C_{j}$ and $K(i)$ is the set of the neighbouring nodes of the vertex $S_{i}$.

\subsection{Steger-Warming flux decomposition}

We describe now the approximation of the term $\int_{\partial C_{i j}} \mathbf{F}\left(\mathbf{Q}_{h}\right) \cdot \nu_{i j} d \sigma$. This integral is evaluated as:

$$
\boldsymbol{\Phi}_{i j}=\widehat{\mathbf{F}}\left(\mathbf{Q}_{h}\right) \cdot \boldsymbol{\eta}
$$


where $\boldsymbol{\eta}=\left(\eta_{1}, \eta_{2}\right)=\int_{\partial C_{i j}} \nu_{i j} d \sigma$ and $\hat{\mathbf{F}}\left(\mathbf{Q}_{h}\right)$ is some approximate value of $\mathbf{F}\left(\mathbf{Q}_{h}\right)$ along $\partial C_{i j}$. We introduce the numerical flux function $\boldsymbol{\Phi}_{i j}$ chosen as a first-order upwind scheme depending on the two states $\left(\mathbf{Q}_{h}\right)_{i}$ and $\left(\mathbf{Q}_{h}\right)_{j}$ on each side of the interface $\partial C_{i j}$. This is a direct extension of the one-dimensional upwind scheme definition of Lax, Harten and Van Leer [2] and more details can be found in [4].

We recall that for the linear case, all first-order upwind schemes reduce to the same [2]. Thus, there is no choice of an upwind scheme for approximating Maxwell equations in homogeneous media. However, in the case of heterogeneous media, discontinuities in the fields may appear at the interface of materials. The way the discontinuities are treated may depend now on the choice of the scheme. We use here the flux decomposition of Steger and Warming [3].

We now introduce the following notations before describing the scheme. The jacobian $\mathcal{A}(\mathbf{Q}, \boldsymbol{\eta})=\boldsymbol{\eta} . \mathbf{F}^{\prime}(\mathbf{Q})$ being diagonalisable, it may be written:

$$
\mathcal{A}=T \Lambda T^{-1}, \Lambda=\operatorname{diag}\left(\lambda_{k}\right)
$$

where $\lambda_{k}$ are the eigenvalues of $\mathcal{A}$ defined by formula (8). One defines $\mathcal{A}^{ \pm}=$ $T \Lambda^{ \pm} T^{-1}$ where $\Lambda^{+}=\operatorname{diag}\left(\max \left(\lambda_{k}, 0\right)\right)$ and $\Lambda^{-}=\operatorname{diag}\left(\min \left(\lambda_{k}, 0\right)\right)$. The numerical flux function of Steger-Warming is given by:

$$
\boldsymbol{\Phi}_{i j}^{S W}=\boldsymbol{\Phi}_{i j}^{S W}\left(\mathbf{Q}_{i}, \mathbf{Q}_{j}\right)=\mathcal{A}^{+}\left(\mathbf{x}_{i}, \boldsymbol{\eta}\right) \mathbf{Q}_{i}+\mathcal{A}^{-}\left(\mathbf{x}_{j}, \boldsymbol{\eta}\right) \mathbf{Q}_{j}
$$

where $\mathbf{Q}_{i}=\mathbf{Q}\left(S_{i}\right), \mathbf{Q}_{j}=\mathbf{Q}\left(S_{j}\right), \mathbf{x}_{i}$ and $\mathbf{x}_{j}$ are the coordinates of nodes $S_{i}$ and $S_{j}$ respectively.

The split matrices in the heterogeneous case are:

$$
\begin{aligned}
& \mathcal{A}^{+}(\mathbf{x}, \boldsymbol{\eta})=\frac{c_{\boldsymbol{r}}(\mathbf{x})|\boldsymbol{\eta}|}{2}\left(\begin{array}{ccc}
\xi_{2}{ }^{2} & -\xi_{1} \xi_{2} & \xi_{2} / Z(\mathbf{x}) \\
-\xi_{1} \xi_{2} & \xi_{1}{ }^{2} & -\xi_{1} / Z(\mathbf{x}) \\
\xi_{2} Z(\mathbf{x}) & -\xi_{1} Z(\mathbf{x}) & 1
\end{array}\right) \\
& \mathcal{A}^{-}(\mathbf{x}, \boldsymbol{\eta})=\frac{c_{\boldsymbol{r}}(\mathbf{x})|\boldsymbol{\eta}|}{\mathbf{2}}\left(\begin{array}{ccc}
-\xi_{2}{ }^{2} & \xi_{1} \xi_{2} & \xi_{2} / Z(\mathbf{x}) \\
\xi_{1} \xi_{2} & -\xi_{1}{ }^{2} & -\xi_{1} / Z(\mathbf{x}) \\
\xi_{2} Z(\mathbf{x}) & -\xi_{1} Z(\mathbf{x}) & -1
\end{array}\right)
\end{aligned}
$$

where $\left(\xi_{1}, \xi_{2}\right)$ is the unit outgoing normal of the cell interface and $Z(\mathbf{x})=\sqrt{\frac{\varepsilon_{r}(\mathbf{x})}{\mu_{r}(\mathbf{x})}}$ or $Z(\mathbf{x})=\sqrt{\frac{\mu_{r}(\mathbf{x})}{\varepsilon_{r}(\mathbf{x})}}$ respectively in the $T M$ and $T E$ case. Taking $\mathbf{Q}_{i}$ and $\mathbf{Q}_{j}$ as constant on each cell, this approach results in a first-order approximation of the fluxes.

\subsection{High-order approximation}

We construct now a third-order accurate scheme in space using the extension of Van Leer's MUSCL method to finite elements $[1,4]$. One way to achieve highorder accuracy is to increase the degree of the solution interpolation in a cell and to 
evaluate the fluxes with some extrapolated values $\mathbf{Q}_{i j}$ and $\mathbf{Q}_{j i}$ at the cell interface $\partial C_{i j}$. This extension requires the evaluation of the gradient of the solution at each node and a modification of the $\boldsymbol{\Phi}_{i j}$ function arguments. The high-order flux function is written:

$$
\left\{\begin{array}{c}
\boldsymbol{\Phi}_{i j}^{S W}=\boldsymbol{\Phi}_{i j}^{S W}\left(\mathbf{Q}_{i j}, \mathbf{Q}_{j i}\right) \\
\mathbf{Q}_{i j}=\mathbf{Q}_{i}+\frac{1}{2}(\vec{\nabla} \mathbf{Q})_{i} . \mathbf{S}_{i} \mathbf{S}_{j} \\
\mathbf{Q}_{j i}=\mathbf{Q}_{j}-\frac{1}{2}(\vec{\nabla} \mathbf{Q})_{j} . \mathbf{S}_{i} \mathbf{S}_{j}
\end{array}\right.
$$

The nodal gradients $(\vec{\nabla} \mathbf{Q})_{i, j}$ may be defined in several ways. One possible choice of definition is the so-called hermitian gradients [5]:

$$
\begin{aligned}
\vec{\nabla} \mathbf{Q}_{i}^{H} & =\frac{1}{\operatorname{Area}\left(C_{i}\right)} \iint_{C_{i}} \vec{\nabla} \mathbf{Q} d x d y \\
& =\left.\left.\frac{1}{\operatorname{Area}\left(C_{i}\right)} \sum_{T, S_{i} \in T} \frac{\operatorname{Aire}(T)}{3} \sum_{k=1}^{3} \mathbf{Q}\right|_{S_{i^{k}}} \vec{\nabla} \varphi\right|_{S_{i k}}(T)
\end{aligned}
$$

where $S_{i^{k}}(k=1,2,3)$ are the three vertices of the triangle $T$ and $\vec{\nabla} \varphi \mid s_{i^{k}}(T)$ the constant gradient on $T$ of the P1 basis function associated to $S_{i^{k}}$.

For accuracy and stability reasons [6], we use the following interpolation:

$$
\left\{\begin{array}{l}
\mathbf{Q}_{i j}=\mathbf{Q}_{i}+\frac{1}{2}\left\{(1-2 \beta)\left(\mathbf{Q}_{j}-\mathbf{Q}_{i}\right)+2 \beta \vec{\nabla} \mathbf{Q}_{i}^{H} \cdot \mathbf{S}_{i} \mathbf{S}_{j}\right\} \\
\mathbf{Q}_{j i}=\mathbf{Q}_{j}-\frac{1}{2}\left\{(1-2 \beta)\left(\mathbf{Q}_{j}-\mathbf{Q}_{i}\right)+2 \beta \vec{\nabla} \mathbf{Q}_{j}^{H} \cdot \mathbf{S}_{i} \mathbf{S}_{j}\right\}
\end{array}, \beta \in(0,1)\right.
$$

This interpolation involves a convex combination of centered slopes $\left(\mathbf{Q}_{j}-\mathbf{Q}_{\boldsymbol{i}}\right)$ and half-upwind ones $\left(\vec{\nabla} \mathbf{Q}_{i, j}^{H} . \mathbf{S}_{i} \mathbf{S}_{j}\right)$ in analogy to the one-dimensional case [7]. Finally, we take $\beta=\frac{1}{3}$ as an upwind parameter in order to achieve a quasi third-order scheme in space for unstructured meshes. In case of structured meshes, this scheme is exactly third-order accurate. Let us note that no limitation technique is used in our high-order scheme since the system is linear in the unknown variables even in the heterogeneous cases treated here.

\section{Material interfaces}

In the presence of dielectric materials, the solution (computed in scattered fields) is also driven by the incident term noted as $\mathbf{S}^{\text {inc }}(5)$. This term, involving both time and space derivatives of the incident wave, is turned into fluxes since the incident wave is assumed to be solution of Maxwell equations in free space. This is a practical way to ensure no contribution of this term in free space (same spatial approximation for the two parts of $\mathbf{S}^{i n c}$ ) and to have an homogeneous approach between the unknowns (scattered fields) and the given incident field. 
Hence, there is no nced to treat apart the material interface with this scheme. One can easily check that the fluxes remain continuous across a material interface independently of the discontinuities of the permittivity and the permeability; the properties of $\mathcal{A}^{+}$and $\mathcal{A}^{-}$insure the discrete Lax jump conditions applied in total fields:

$$
\left.\begin{array}{l}
{[\mathbf{n} \wedge \mathbf{E}]=0} \\
{[\mathbf{n} \wedge \mathbf{H}]=0}
\end{array}\right\} \Longleftrightarrow \boldsymbol{\Phi}_{i j}^{S W}+\boldsymbol{\Phi}_{j i}^{S W}=0
$$

\section{Boundary conditions}

The terms $<2>$ and $<3>$ of the right-hand-side of (14) contain the physical boundary conditions which are usually expressed in terms of electric and magnetic fields $(\mathbf{E}, \mathbf{H})$.

Perfectly conducting surface:

On the surface $\Gamma_{b}$, the field is assumed to satisfy the condition (9). Thus, the boundary term $<2>$ is computed as:

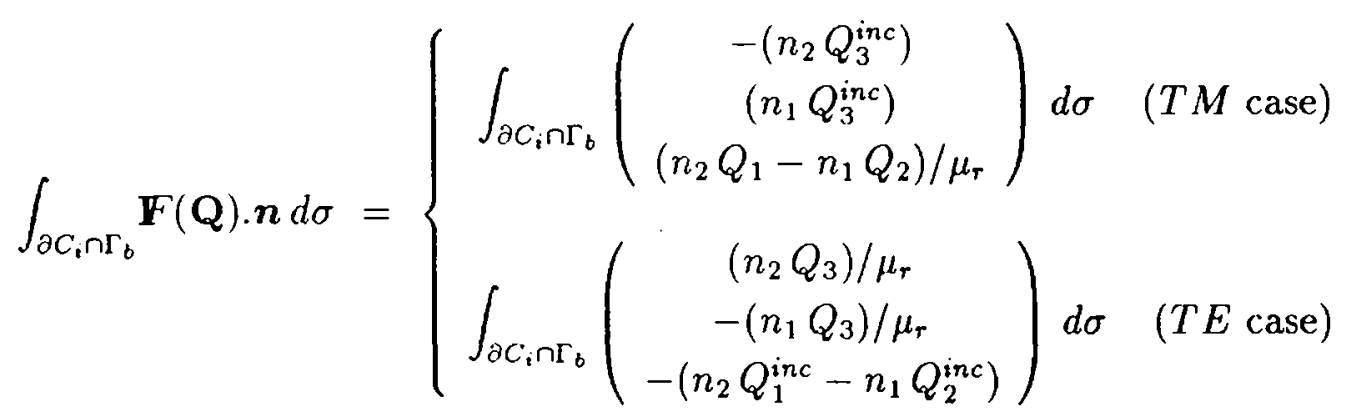

where $Q_{l, l=1 \ldots 3}$ are the components of the computed field $\mathbf{Q}$ on $\Gamma_{b}, \mathbf{Q}^{\text {inc }}$ is the given incident field and $\boldsymbol{n}=\left(n_{1}, n_{2}\right)$ is the outward normal to $\Gamma_{b}$. Let us note that in this procedure, the boundary condition (9) is applied in a weak form.

\section{Absorbing condition :}

The artificial boundary $\Gamma_{\infty}$ is assumed to be set in free space. The first-order boundary condition of Silver-Müller (10) then reduces for both $T E$ and TM cases to the following equation:

$$
Q_{3}=n_{2} Q_{1}-n_{1} Q_{2}
$$

The above condition may be inserted, as it is, into the term $<3>$ which becomes:

$$
\int_{\partial C_{i} \cap \Gamma_{\infty}} \mathbf{F}(\mathbf{Q}) \cdot \boldsymbol{n} d \sigma=\int_{\partial C_{i} \cap \Gamma_{\infty}}\left(n_{2} Q_{1}-n_{1} Q_{2}\right)\left(\begin{array}{c}
n_{2} \\
n_{1} \\
1
\end{array}\right) d \sigma
$$

Here again, the boundary condition is satisfied in a weak form only.

One may also use an upwinding which sorts automatically between the in- and out-going informations and then selects the right boundary condition to be considered. In our case (Maxwell system), this condition is associated to waves which 
travel at speed $-c$. As it was done inside the computational domain $\Omega$, we choose the Steger-Warming scheme on the boundary $\Gamma_{\infty}$ which is written now:

$$
\boldsymbol{\Phi}_{i \infty}^{S W}=\boldsymbol{\Phi}^{S W}\left(\mathbf{Q}_{i}, \mathbf{Q}_{\infty}, \boldsymbol{n}\right)=\mathcal{A}^{+} \mathbf{Q}_{i}+\mathcal{A}^{-} \mathbf{Q}_{\infty}
$$

where $\mathbf{Q}_{\boldsymbol{i}}$ is the computed scattered field on $\Gamma_{\infty}$ and $\mathbf{Q}_{\infty}$ is the scattered field outside the domain $\Omega$. Assuming that the scattered field is zero outside the domain, one gets:

$$
\Phi_{i \infty}^{S W}=\mathcal{A}^{+} \mathbf{Q}_{i}
$$

This expression may be viewed as an absorbing condition (no information entering the domain). Furthermore, one can easily check that the two ways the boundary condition on $\Gamma_{\infty}$ is considered are equivalent. Indeed, using the relation (16), one may rewrite the formula (17) as:

$$
\int_{\partial C_{\mathfrak{i}} \cap \Gamma_{\infty}} \mathbf{F}(\mathbf{Q}) \cdot \boldsymbol{n} d \sigma=\frac{1}{2} \int_{\partial C_{\boldsymbol{i}} \cap \Gamma_{\infty}}\left(n_{2} Q_{1}-n_{1} Q_{2}+Q_{3}\right)\left(\begin{array}{c}
n_{2} \\
n_{1} \\
1
\end{array}\right) d \sigma
$$

The latter formula gives explicitely the flux $\Phi_{i \infty}^{S W}$. One may conclude that using an upwind scheme at the outer boundary corresponds to a first-order absorbing condition.

\subsection{Time integration}

The above discretisation leads to the semi-discrete system associated to each node $S_{i}$ (the subscripts $S_{i}$ and $h$ are dropped):

$$
\mathbf{Q}_{\tau}+\Psi(\mathbf{Q})=0
$$

where $\boldsymbol{\Psi}$ contains the space discretisation and incident wave terms.

We need an accurate time integration when dealing with unstationary problems. The most simple and efficient way to construct a third-order accurate scheme is to use a three-step explicit Runge-Kutta method. Besides, this method requires little storage and fits perfectly to massive parallelism. It goes as follows:

$$
\left\{\begin{array}{l}
\mathbf{Q}^{0}=\mathbf{Q}^{n} \\
\mathbf{Q}^{l}=\mathbf{Q}^{0}-\frac{\Delta \tau}{(4-l)} \boldsymbol{\Psi}\left(\mathbf{Q}^{l-1}\right) \quad l=1,2,3 \\
\mathbf{Q}^{n+1}=\mathbf{Q}^{3}
\end{array}\right.
$$

\subsection{Radar Cross Section evaluation}

The method presented above solves Maxwell equations in a finite domain and we get the time-dependent scattered field when an object is illuminated by an incident plane wave. This is the near-ficld solution while the bistatic Radar Cross Section 
(RCS) evaluation requires the computation of the scattered field intensity at infinity in the frequency-domain. The bistatic RCS is defined in two dimensions as:

$$
\sigma=\lim _{r \rightarrow \infty} 2 \pi r \frac{\left|{\widehat{Q_{3}}}^{s}\right|^{2}}{\left|{\widehat{Q_{3}}}^{i n c}\right|^{2}}
$$

where $\left|{\widehat{Q_{3}}}^{\text {inc }}\right|$ is the amplitude of the incident wave and ${\widehat{Q_{3}}}^{s}$ the Fourier component of the time-dependent solution [16]. $Q_{3}$ refers to the variable $E_{z}$ or $H_{z}$ whether we consider the $T E$ or $T M$ mode.

To get a time-harmonic solution, calculations are pursued until the fields reach a sinusoidal steady state depending on the frequency of the incident wave. Convergence comes from the limiting amplitude principle [18] and the complex field in the frequency-domain is computed from the time-history of the solution by using a Fourier transform. The far-field solution then comes via an asymptotic approach using the Green function. From the theory of integral equations and an asymptotic expression of the Hankel functions, one can get the following far-field representation $[16,19]$ :

$$
\tilde{U}(r, \phi, U) \simeq \sqrt{\frac{2}{\pi k r}} \mathrm{e}^{-i \pi / 4} \mathrm{e}^{i k r} P(k, \phi, U)
$$

with:

$$
P(k, \phi, U)=\frac{-i}{4} \int_{S}\left(\frac{\partial U}{\partial \mathbf{n}_{\mathbf{s}}}+i k\left(\mathbf{n}_{\mathbf{s}} \cdot \mathbf{e}\right) U\right) \mathrm{e}^{-i k r_{s} \mathbf{e}_{\mathbf{s}} \cdot \mathbf{e}}\left(x_{s}\right) d \sigma\left(x_{s}\right)
$$

where $S$ is a surface (generally taken in free space) around the body, $r_{s} \mathbf{e}_{\mathbf{s}}$ the coordinate-vector of a surface point, $r$ e (or $(r, \phi)$ in polar coordinates) the coordinatevector of a point at infinity and $k$ is the wave number.

For an incident plane wave of unit amplitude, the RCS may be finally written as:

$$
\sigma=\frac{4}{k}\left|P\left(k, \phi, Q_{3}\right)\right|^{2}
$$

\subsection{Convergence to the steady state}

When we approximate periodic solutions of Maxwell system (for RCS calculations for instance) using a time-marching method, one should consider the following points:

(a) ensure that the steady state is well reached.

(b) ensure that no extra-calculations have been done.

(c) how to accelerate the convergence while maintaining a high accuracy in time.

The latter point is nowhere here considered. Points (a) and (c) may be summed up in defining a good convergence criterion.

We used first the criterion proposed in [13] with a perfectly conducting cylinder test case. The solution is then supposed to have reached the periodic state when it 
is of the form:

$$
\mathbf{Q}(\mathbf{x}, \tau)=\mathbf{Q}_{0}(\mathbf{x}) \cos \left(k \tau+\phi_{0}\right)
$$

where $k=\frac{\omega}{c}$ is the wave number of the incident field. Such a solution verifies the differential equation in time variable:

$$
\ddot{\mathrm{Q}}=-k^{2} \mathbf{Q} \quad(*)
$$

The discretisation of $(*)$ allows to define the following criterion:

$$
L O^{n}=\frac{\left\|\left(\delta_{t} Q_{3}+k^{2} d t^{2} Q_{3}\right)(\mathbf{x}, \lambda+(n-1) d t)\right\|_{L_{2}}}{\left\|\left(\delta_{t} Q_{3}+k^{2} d t^{2} Q_{3}\right)(\mathbf{x}, \lambda)\right\|_{L_{2}}}, n \geq 1
$$

We performed, on the same test case, calculations with two different values of the time step: $\mathrm{dt}$ and $2 \mathrm{dt}$. The corresponding time-histories denoted $\mathrm{LO}(\mathrm{dt})$ and $\mathrm{LO}(2 \mathrm{dt})$ respectively are plotted on Figure 2. We observe that the $\mathrm{LO}(\mathrm{dt})$ residual reaches a value of an order of magnitude less than the second one $\mathrm{LO}(2 \mathrm{dt})$. The observed behaviour of this residual shows that the criterion LO is dependent on the time step. Indeed, reducing the time step leads to a better approximation of $\left(^{*}\right)$. However, this seems to be in opposition to points (b) and (c).

We suggest here a criterion based on the energy as a test of convergence. This test is performed either on the transverse electric field or on the transverse magnetic one using the $L_{2}$ norm of the scattered field. The energy is evaluated on the whole domain at each period in time:

$$
E 1^{n}=\frac{\left|\left(\left\|Q_{3}(\mathbf{x}, n \lambda)\right\|-\left\|Q_{3}(\mathbf{x},(n-1) \lambda)\right\|\right)\right|}{\left|\left(\left\|Q_{3}(\mathbf{x}, \lambda)\right\|-\left\|Q_{3}(\mathbf{x}, 0)\right\|\right)\right|}, n \geq 1
$$

We performed the calculations until the residue $E 1$ reached some fixed value $\left(10^{-3}\right.$ in this case). The RCS solution obtained at this stage compared very well with the exact one but we noticed that the RCS solution was already accurate some time steps earlier. Thus, many more operations than needed were performed.

Finally, we suggest to improve the criterion $E 1$. This is achieved by evaluating the $L_{2}$ norm in a convex domain including the illuminated body and delimited by the surface where the RCS calculation is performed $(E 2)$. Now, errors due to the artificial boundary affect no more the value of the residue. It is meaningful since only local informations around the body are needed for RCS evaluation.

The three criteria $L O, E 1$ and $E 2$ are compared in Figure 3. E2 is the criterion which senses the steady state the better. Besides, we did not observe any dependency on the time step for this one.

This criterion is costless in memory storage and time, and seems to be well adapted to time-periodic simulations as far as the numerical method converges. It seems that time-marching methods could not be very efficient to solve scattering problems involving non convex bodies. In this case, the trapped rays (in inlets for example) may affect the convergence drastically. Solutions do not any more converge at exponential speed to the periodic steady state. Some authors suggested methods to accelerate the convergence either by enforcing the periodic state through a minimisation technic [14] or by introducing the frequency of the incident wave in the scheme [22]. 


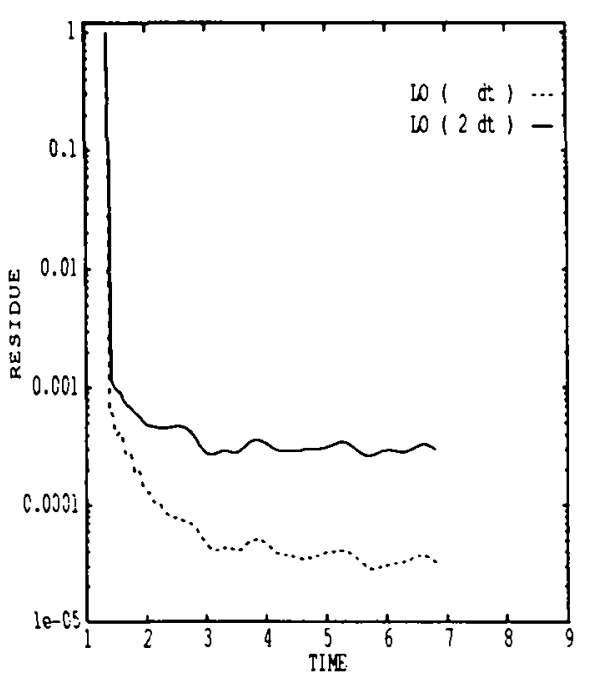

Figure 2: Sensibility of the criterion $L O$ to the time step

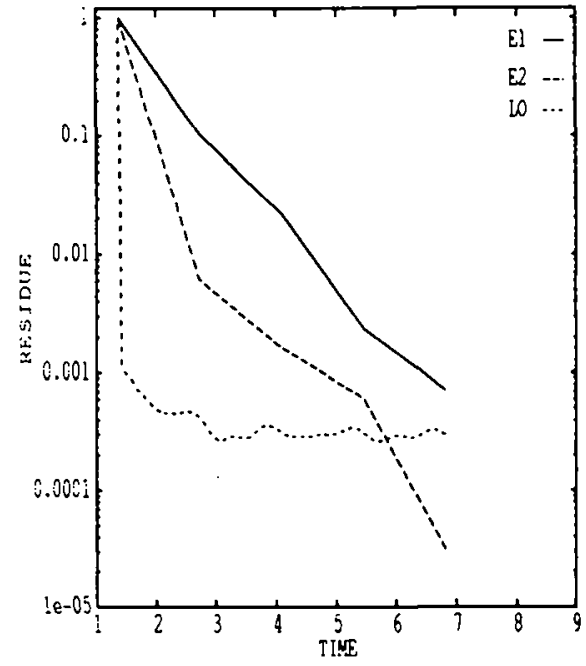

Figure 3: Comparison of the three criteria

\subsection{Divergence error}

We do not consider the divergence-free conditions in our model, since they are redundant in the continuous equations. Numerically, we only take these equations into account at the initial time. We use piecewise linear functions in our scheme, obviously not satisfying these conditions. Many authors using Finite-Element methods in electromagnetics make sure of these conditions by using a space of divergence-free discrete functions [20] or more recently using a divergence correction method [15].

Our numerical approximation is not based on a Finite-Element method but a Finite-Volume one which belongs to the class of conservative schemes. Piecewise linear functions are used here to interpolate the fields at the cell interfaces. Although it is well known that these schemes are appropriate to treat divergence terms, nevertheless we look at the conservation of the divergence-free property by our method. We refer to [13] for a similar study on FCT methods.

Unstructured grids do not yield an unic way to discretize the divergence. We evaluate this divergence in a weak way and we compare it for three different size grids where a square body is illuminated by an incident plane wave. At each time step, we consider the $L_{\infty}$ norm of the divergence of the solution. The divergence reaches quickly a periodic state whose average amplitude is numerically observed to be $O(h)$ where $h$ is the mesh step (Fig. 4). Furthermore, one can notice the great influence of the space accuracy of the scheme on the divergence: the amplitude is reduced by a factor five when comparing a first-order and a quasi third-order scheme in space (Fig. 5). We can conclude that the Finite-Volume method used with unstuctured meshes does not conserve exactly the divergence-free conditions and depends essentially on the mesh thickness as well as on the accuracy of the scheme. However, the good accuracy of the RCS calculations (section 4) obtained 
with meshes corresponding to 12 up to 20 points per wavelength shows that the distortion of the divergence observed with unstructured meshes has no significant influence on the solution.

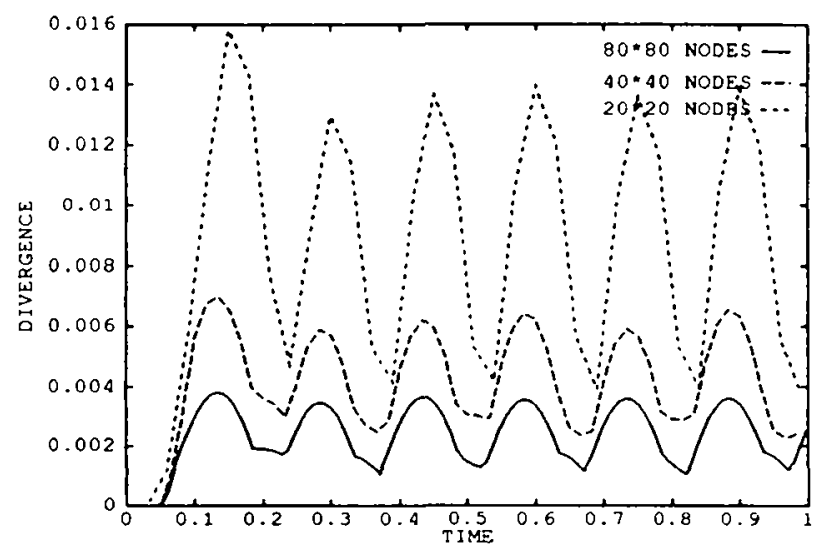

Figure 4: Size grid comparison

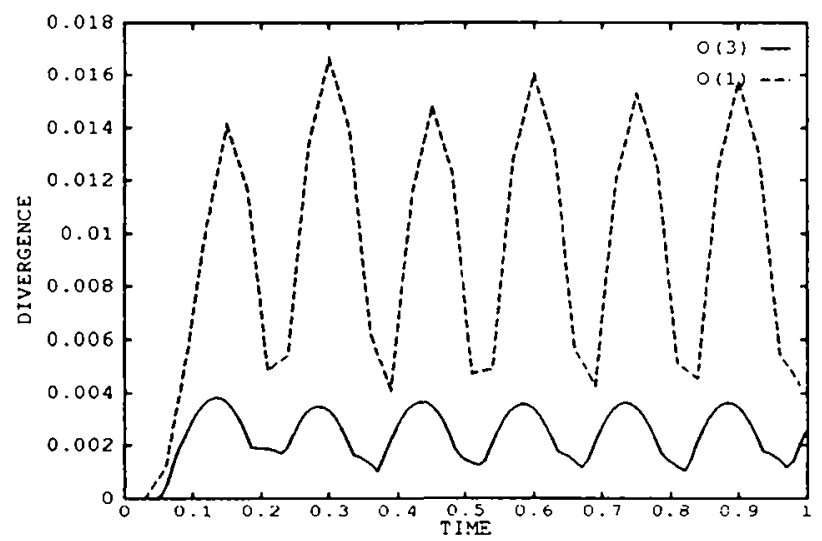

Figure 5: First- and third-order scheme comparison

\section{Numerical experiments}

\subsection{Unstationary experiments}

We simulated two scattering problems of a pulse impinging on a metallic cylinder of circular or square section.

A continuous $T M$ incident wave whose electric field is of the form:

$$
E_{z}^{i n c}=\cos \{|\mathbf{k}|(x \cos \phi+y \sin \phi-c t)\}
$$

is taken on half a wave length and set to zero elsewhere. The expressions of $H_{x}^{\text {inc }}$ and $H_{y}^{\text {inc }}$ are deduced from Maxwell equations:

$$
H_{x}^{i n c}=\frac{\sin \phi}{\mu c} E_{z}^{i n c}, H_{y}^{i n c}=-\frac{\cos \phi}{\mu c} E_{z}^{i n c}
$$


where $\phi$ is the angle of incidence. The initial time is chosen just before the pulse encounters the body and the wave is coming from the right side.

Figure 7 shows a good behaviour of the total field solution. The incident wave is perturbed near the body in order to satisfy the boundary conditions. One can distinguish very well the scattered wave on the right side. No deterioration of the solution appears at the corners of the square section cylinder.

\subsection{Time-harmonic results}

Some bistatic RCS simulations are presented for infinite circular cylinders and compared with exact solutions [17]. TE and $T M$ results are shown both in perfectly conducting and coated cases.

The bodies are illuminated by a continuous harmonic incident wave and the calculations are performed until the solution reaches a sinusoidal steady state. We use a criterion based on the energy (section 2). We compute only the real part of the solution and get the complex field representation through a Fourier Transform. The RCS response is normalized by the wave length and plotted on a logarithmic scale:

$$
\sigma=10 * \log _{10}\left(\frac{R C S}{\lambda}\right)
$$

We represent the RCS in $\mathrm{db}$ as a function of the viewing angles $\theta$ where the value $180^{\circ}$ of $\theta$ always represents the backscatter point. The case of a metallic cylinder surrounded by the vaccum is presented on Figure 9 such that $k a_{0}=10\left(k=\frac{2 \pi}{\lambda}\right)$ where $\mathrm{k}$ denotes the wave number and $a_{0}$ the radius. One can see a good agreement with the exact solution for both $T M$ and $T E$ cases with a mesh (Fig. 8) corresponding to 20 points per wavelength near the body.

Two $T E$ cases are also presented for the previous metallic cylinder but coated with a lossless dielectric of thickness $\left(a_{1}-a_{0}\right)$. We use the same mesh as in the previous case (Fig. 8) with $\varepsilon_{r}=2.56$ and $\mu_{r}=1$ in the coating. We have in the first case (Fig. 10) $k a_{1}=7.7$ and $k a_{0}=7$. One can notice the good accuracy of the solution and the ability of the solver to treat coated materials and field discontinuities. The solution of Fig. 11 is the same test case with a higher frequency ( $k a_{1}=11, k a_{0}=10$ ). The numerical solution compares well with the exact one despite the numerous variations. However, the response is not so accurate as in the previous examples. This is probably due to the coarseness of the mesh used since the number of points per wavelength in the dielectric coating is here about 12. This number (12) seems to be a minimum, because the solution would not be accurate enough with less points according to the parameters of this test case. 
Figure 7: Time-evolution of the total field $E_{z}$
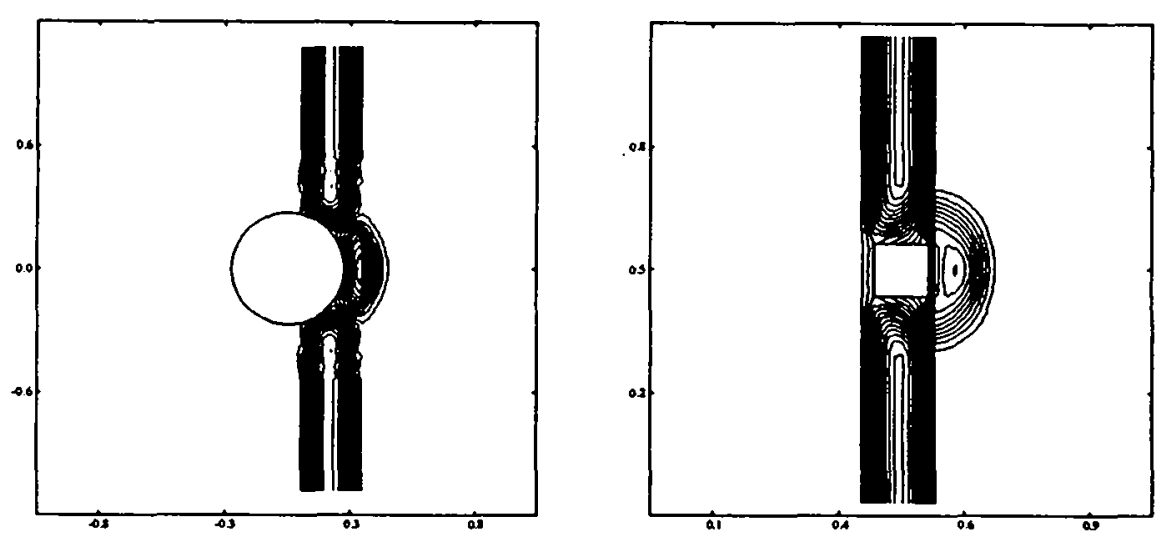

$\tau=0.25$

$\tau=0.15$
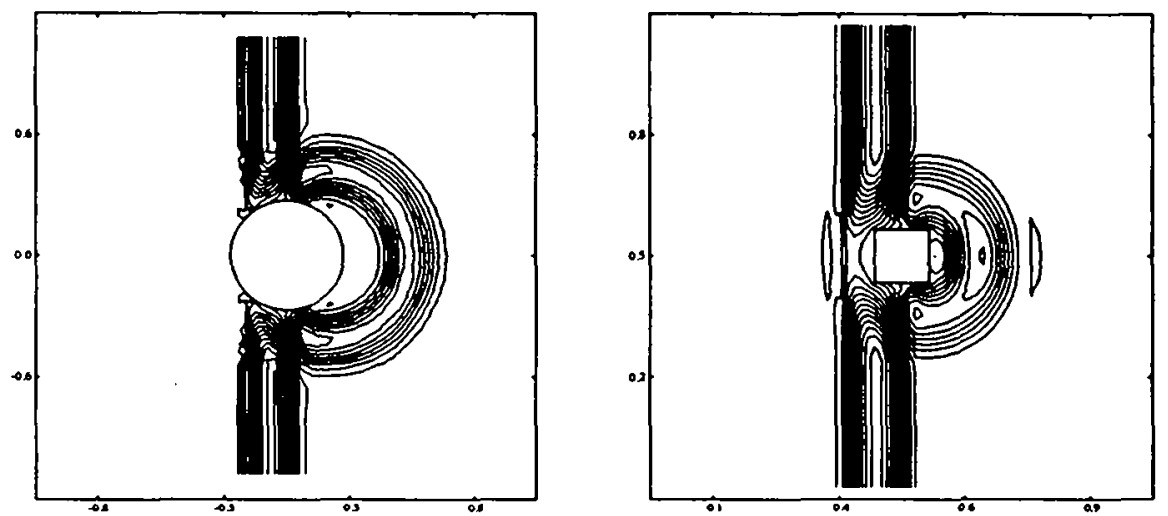

$\tau=0.50$

$\tau=0.20$
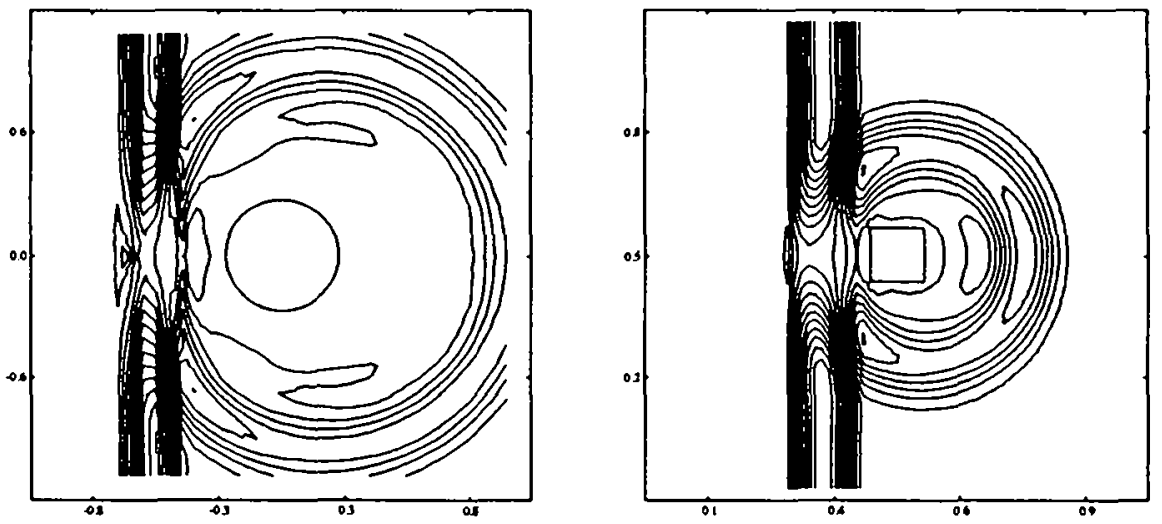

$\tau=1.00$

$\tau=0.30$ 


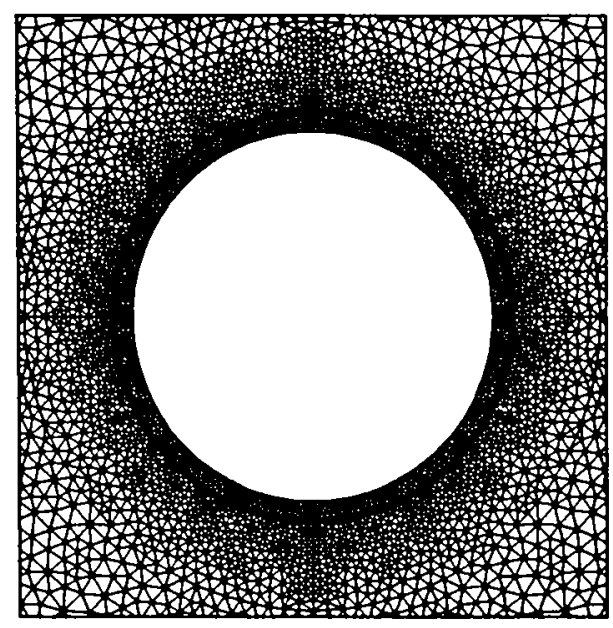

Figure 8: Partial view of the mesh

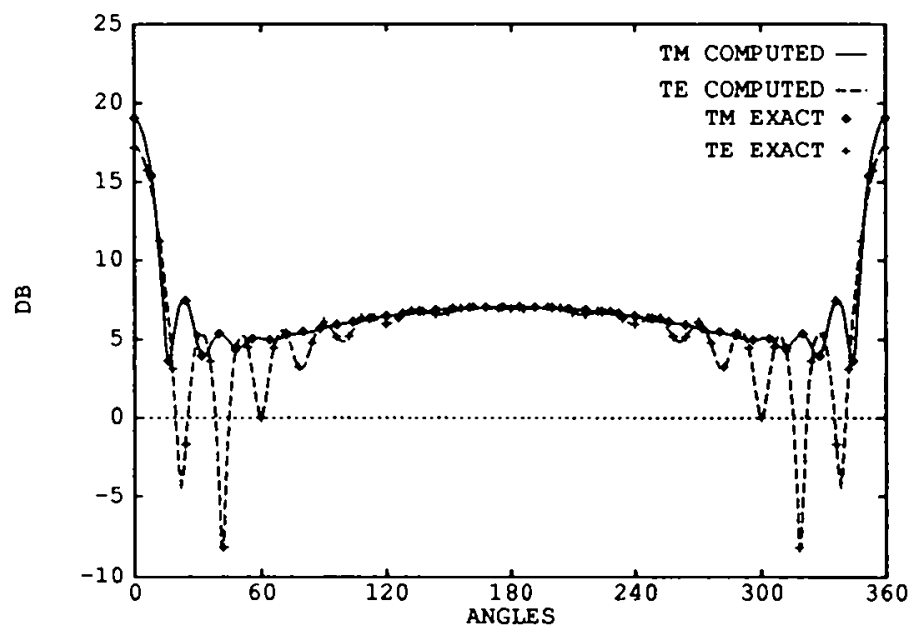

Figure 9: $T E$ and $T M$ cases, $k a_{0}=10$ ( perfectly conducting cylinder) 


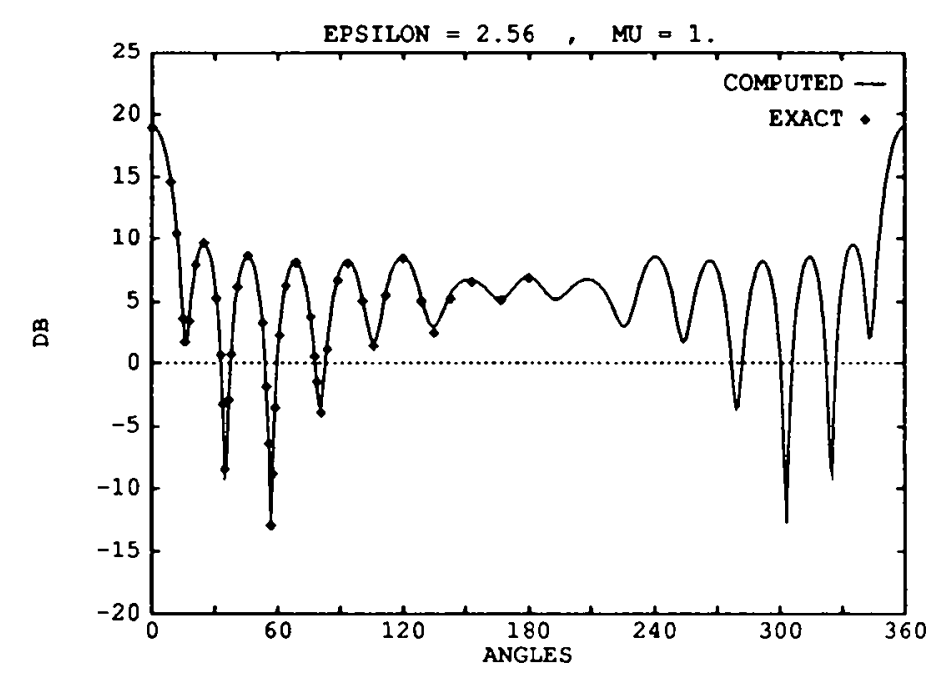

Figure 10: $T E$ coated case, $k a_{0}=7, k a_{1}=7.7$

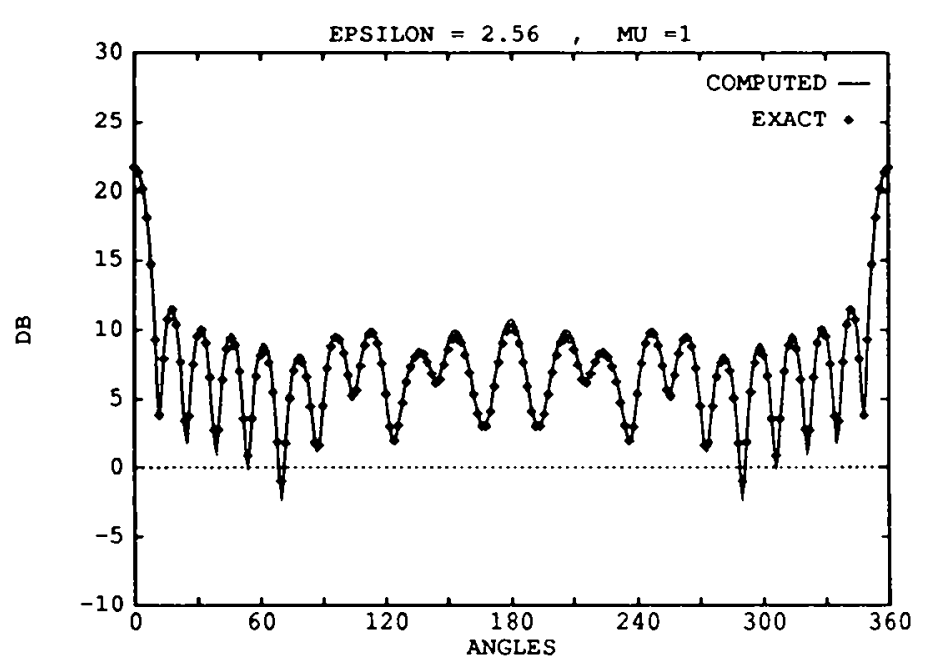

Figure 11: $T E$ coated case, $k a_{0}=10, k a_{1}=11$

The series of the previous examples is interesting since exact solutions are known. The next experiment is less academic.

A metallic NACA0012 is illuminated by a transverse magnetic plane wave at incidence $\phi=90^{\circ}$. The body is four wave lengths long and the frequency of the incident wave is $1.2 \mathrm{Ghz}$. It corresponds to a number $k a_{0}=12.57$. The number of points per wave length is on average around 15 near the body. Figure 12 depicts the scattered electric field. The solution in the backscatter area has a wave front which corresponds very well to the geometry of the body. In the forward area, the solution is in opposition with the incident field. This is clearly seen on Fig. 13 showing the total field. This corresponds to the shadow region. 
We can notice that the RCS diagram (Fig. 14) is oscillation free. In contrast with the previous examples, we can observe two maxima for the viewing angles $\theta=0^{\circ}$ and approximately $\theta=180^{\circ}$. The first one corresponds to a receptor placed in front of the incoming wave. A large amount of energy has travelled along this direction. The second one which was not observed with the circular cylinders is due to the flatness of the body. The rays are little diffracted in the backscatter area.

The RCS is compared on the same figure (Fig. 14) with a numerical integral method solution [21]. The mesh step used was of $\lambda / 50$ on the surface with the integral method while it was of $\lambda / 40$ near the body in our simulations. The difference is less than $0.5 \mathrm{db}$ regarding the whole range of the viewing angles. This gives an excellent agreement between the two solutions.

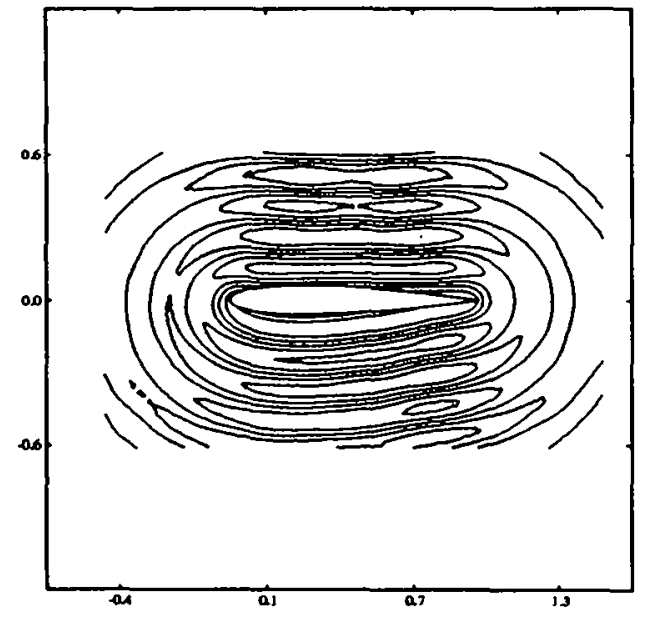

Figure 12: Scattered electric field Min $=-1.11, \operatorname{Max}=0.89$, Delt $a=0.4$

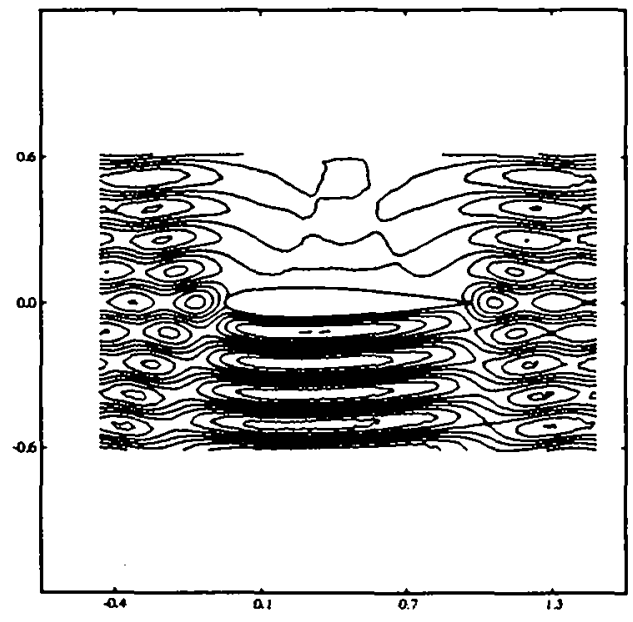

Figure 13: Total electric field $\operatorname{Min}=-1.98, \operatorname{Max}=2.02$, Delta $=0.4$

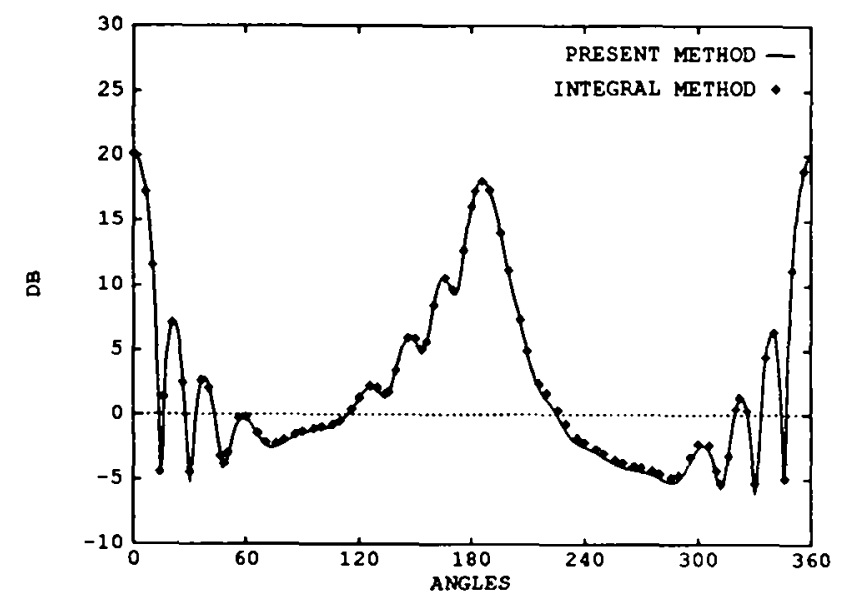

Figure 14: RCS with an incidence angle $\phi=90^{\circ}$ 


\section{Parallel implementation on the Connection Ma- chine}

\subsection{Presentation of the CM2/200}

We first recall the main features of the Connection machine CM2 [26] before describing the parallel algorithm.

The CM2 is a SIMD (Single Instruction Multiple Data) distributed memory machine containing up to $64 \mathrm{~K}(\mathrm{~K}=1024)$ bit-serial processors having their own memory. These processors are arranged 16 to a chip in a hypercube topology (of dimension 12 for the $64 \mathrm{~K}$ configuration). Each weitek (a pair of chips) may be supported by a 32 or 64-bit floating point accelerator (FPA) which increases floating point calculations rate by a factor of 20 . The weitek clock speed is $7 \mathrm{MHz}(10 \mathrm{Mhz}$ for the CM2/200). Mainly two communication forms can be provided from the Connection machine:

- ROUTER: The general mechanism (ROUTER) allows parallel accesses among the distributed memory so that any processor can access the memory of any other processor. General communications use the router network which connects the chips on the hypercube.

- NEWS: The NEWS (North-East-West-South) network is a structured mechanism which optimizes grid communications by exploiting the four nearest neighbours connections. NEWS network is much faster than the ROUTER one but requires regular patterns.

In addition to the Paris execution model available on all CM2 models, the Connection Machines supported by 64 -bit FPA (the CM2/200 is one of them) provide the slicewise model [27] through CM-Fortran compiler switches. These two models are source-code-compatible although the organisation of the memory is drastically different.

The virtual processing facility of the Connection Machine allows the use of more processing nodes than it is physically available. This is achieved through a splitting of the local memory. Each physical processing node simulates some number of virtual processing nodes and the ratio between the virtual ones to the physical ones is called VPR. The two models execute virtual processing in a similar way but the number of the physical processing nodes is different for a given machine-size. Thus, the slicewise model does not require a power of two VPR like in Paris; one can reach high values of VPR quite easily and exploit in a better way the capabilities of the CM2/200 while using less memory.

The main improvements are that elemental computations requiring no communication can be speeded up noticeably and less memory is used. However, this enhancement in performance may only be obtained using the CM-Fortran language which offers a large set of tools for array computations. 


\subsection{Description of the algorithm}

The numerical method described in section 2 leads to the following serial algorithm:

For each time-step :

Step A1 : Compute the nodal gradients

Step B1 : Compute the interpolated values and fluxes

Step C1 : Update the solution at nodes

Step A1 involves computations on neighbouring triangles on each node. Step B1 computes and gathers quantities on neighbouring edges while step $\mathrm{Cl}$ contains nodal computations.

One can notice that the three steps require the manipulation of three classes of objects: triangles, edges and nodes. Several choices of parallel data structure have been analyzed in [24] for the implementation of a CFD code on the CM2; it was shown that the vertex-based parallel structure including nodes and geometric neighbouring node data is the most appropriate for the mixed Finite-Volume/Finite-Element method. This distribution has been used in this study. The parallel algorithm then consists of the three steps $\mathrm{A} 1, \mathrm{~B} 1, \mathrm{Cl}$ completed by the communication phases:

Step A0 : Communicate the neighbour states.

Step B0 : Communicate the neighbour gradients.

This choice is a compromise in the sense that it minimizes the amount of communications but leads to redundant computations in steps A1 and B1.

The use of unstructured meshes yields non-regular patterns for communications. As NEWS-network cannot be used in this case, we optimize the inter-processor communications by using a communication compiler of the system. This communication compiler (Fastgraph) consists in storing and optimizing the message paths so that address calculations are performed once for all time steps. However, this requires the mesh to be static which is our case throughout this study. A mapping technic has been associated with the compilation of the communication graph in order to find a good projection of the mesh nodes onto the hypercube [25].

\subsection{Performance results and comparisons}

We present in this section several performance results of the Maxwell solver obtained on the Connection Machine 8K-CM2/200. Both simple and double precision results are compared with the CRAY YMP mono-processor (64-bit precision) results as well as with the $8 \mathrm{~K}-\mathrm{CM} 2$ ones (32-bit precision).

The algorithm implemented on the CRAY was highly vectorized by a colouring technic on edges and triangles of the meshes. All the measures were plotted for a set of four unstuctured meshes consisting of $8 \mathrm{~K}$ up to $64 \mathrm{~K}$ nodes.

We first evaluate the percentage of communication time over CPU time (Fig. 15) under the slicewise execution model (CM2/200, 32-bit precision). The main part of the CPU time (80\%) is spent on communicating when no communication compiler 
is used (with or without mapping). Address calculations form the most expensive part of the communication phase when dealing with an irregular graph. It does not depend significantly on the way the nodes are mapped onto the hypercube. The best results are obtained when using a good mapping together with the communication compiler (FG as Fastgraph) whose contribution is very important. Finally, we can reduce the percentage of communication time to about $20 \%$.

Different Connection Machines and execution models are compared in single precision (Fig. 16). Executing under the paris model on the CM2/200 allows us to win about $30 \%$ in time compared to the $\mathrm{CM} 2$ results; this corresponds exactly to the increase of the clock speed on the CM2/200 (10 Mhz against $7 \mathrm{Mhz}$ ). We can also measure the efficiency of the slicewise model: we get more than a factor 2 with the CM2 in favour of the CM2/200.

The last results are comparisons between the CRAY YMP and the CM2/200. Although single precision results under paris (Fig. 17) are of the same order than the ones of the CRAY, the double precision is still costly. Concerning the slicewise model, the CM2/200 is faster than the CRAY in any case (Fig. 18).

Particularly, the $8 \mathrm{~K}-\mathrm{CM} 2 / 200$ (32-bit precision) is almost twice faster than the CRAY YMP mono-processor which deals at least with 64 -bit precision. Nevertheless, single precision was enough to obtain accurate results for the test cases presented in this paper.

In the next figures, the letters S, P, SP, DP refer respectively to Slicewise model, Paris model, Simple Precision and Double Precision.

VPR denotes the ratio (user's nodes) $/ 8 \mathrm{~K}$.

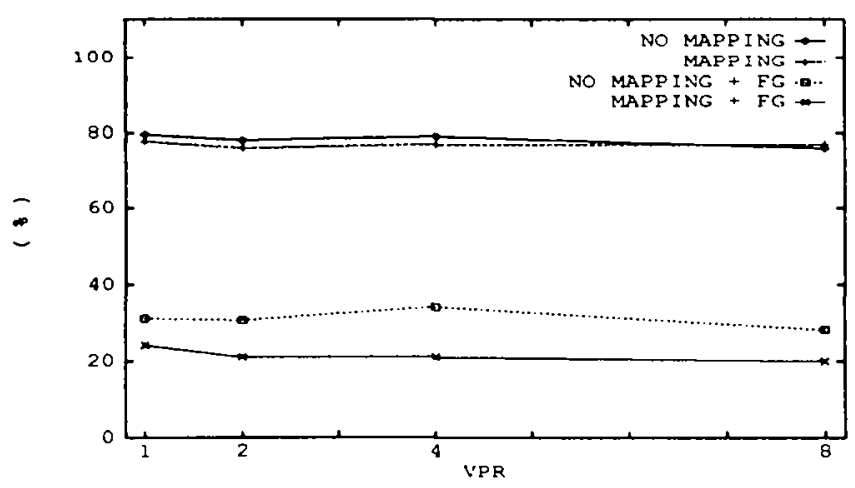

Figure 15: Percentage of communication time / CPU time 


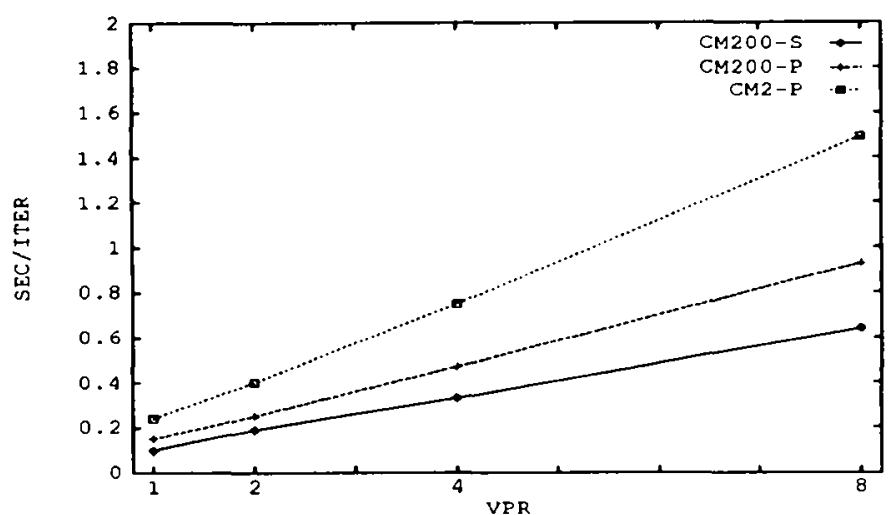

Figure 16: CPU time / iteration (32-bit precision)

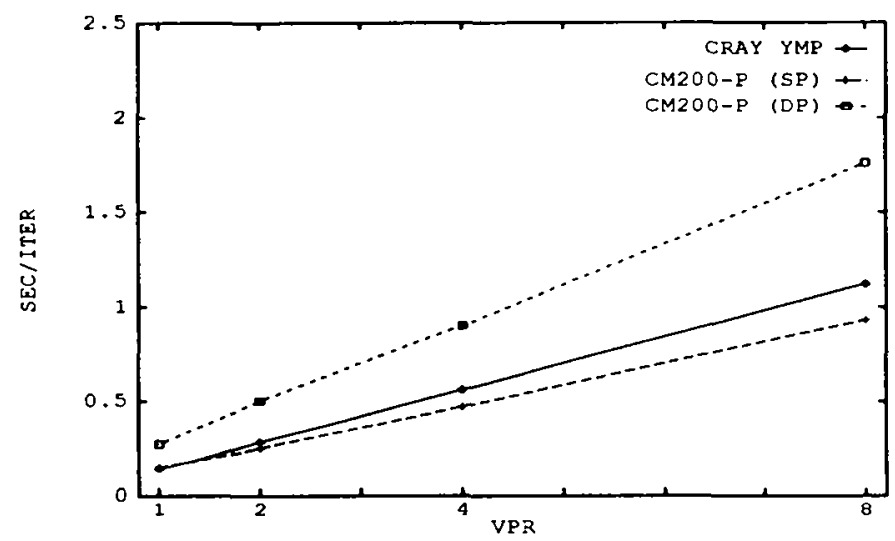

Figure 17: CPU time / iteration (paris model)

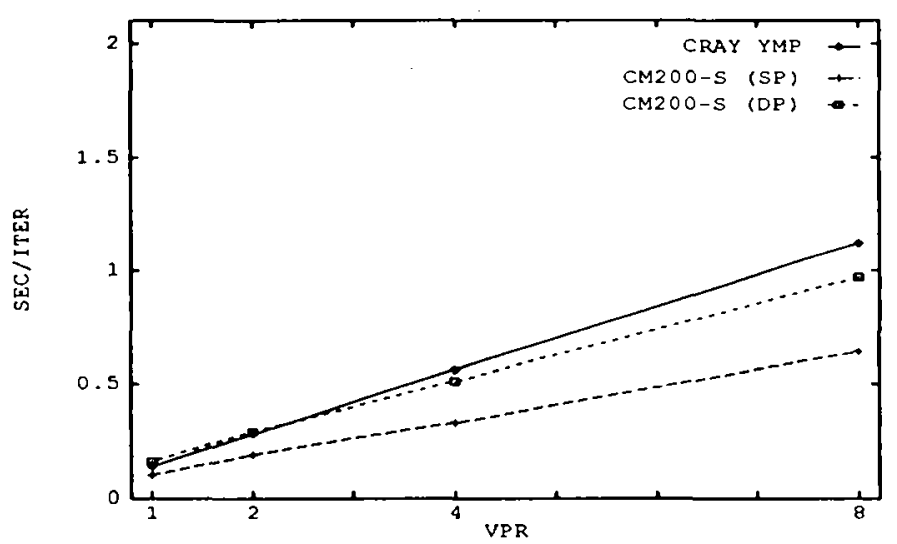

Figure 18: CPU time / iteration (slicewise model) 
The performances of the solver in Mflops (millions of operations per second) are reported in Table 1 both for the CRAY YMP mono-processor and the $8 \mathrm{~K}$-CM2/200. We point out that the redundant operations are not counted in the evaluation of the performance (in Mflops). Indeed, this is unfair to the Connection Machine since the redundant operations (which are not necessary for the obtention of a solution) are effectively done.

\begin{tabular}{|c|c|}
\hline Machine & Mflops \\
\hline \hline CRAY YMP (64-bit) & 113 \\
\hline 8K-CM2/200 (64-bit) & 83 \\
\hline 8K-CM2/200 (32-bit) & 124 \\
\hline
\end{tabular}

Table 1: Performances in Mflops

However, the performance results on the $8 \mathrm{~K}-\mathrm{CM} 2 / 200$ in single precision (32-bit) are better than those obtained on the CRAY YMP (64-bit). Nevertheless, they are not so satisfying regarding the real peak rate of this Connection Machine, which is evaluated to be around 1.5 Gflops. One may explain this loss of performance by two main reasons:

- $20 \%$ of the CPU time is spent on communications between processing nodes (Fig. 15). This is due to the use of irregular patterns on hypercube topology.

- redundant operations are not considered in the evaluation of the Mflops although they are numerous.

However, the performance results (in terms of CPU time and Mflops) show that the present Maxwell solver runs efficiently both on a vector and on a massively parallel computer.

\section{Conclusion}

We developed a time-domain Maxwell solver using a method coming from Computational Fluid Dynamics. We used an accurate upwind scheme in a FiniteVolume formulation with unstructured meshes and proved its efficiency to treat coated materials. The good comparisons of the computed solutions with the exact ones, whenever possible, show that this solver is reliable. Moreover, the method presented compares very well with an integral method in the harmonic case. It is not necessary to consider the divergence-free equations with this conservative scheme. Besides, this solver does not involve any particular technic to treat the field discontinuities occuring at material interfaces.

A massively parallel version of the algorithm has been implemented on the Connection Machine. The main performance results show that a $8 \mathrm{~K}-\mathrm{CM} 2 / 200$ is almost twice faster than a CRAY YMP mono-processor (in terms of CPU time) for this type of application involving irregular communication graphs. We think that the parallel code is well optimized, as the communication time seems hardly reducible. The extension of this solver to more general cases, such as anisotropic media, electric 
or magnetic losses, seems straightforward and will be investigated. This solver also extends directly to the three-dimensional case. Moreover, this time-domain parallel solver may be more useful for some more genuine instationary problems than the scattering of pulses, such as simulations of charged particule beams in electomagnetic fields.

\section{Acknoledgments:}

The authors would like to thank Armel de la Bourdonnaye, Stéphane Lantéri and Jacques Périaux for helpful comments and suggestions.

\section{References}

[1] VAN LEER B., Towards the ultimate conservative difference schemes $V$ : a second order sequel to Godunov's method, J. Comp. Phy., Vol 32 (1979)

[2] LAX P.D. - HARTEN A. - VAN LEER B., On upstream differencing and Godunov type schemes for hyperbolic conservation laws, SIAM Revue, Vol 25, No 1 (1983).

[3] STEGER J. - WARMING R.F., Flux vector splitting for the inviscid gas dynamic with applications to finite-difference methods, J. Comp. Phy., Vol 40, pp. 263-293 (1981)

[4] FEzOUi L. - STOUfFlet B., A Class of Implicit Upwind Schemes for Euler Simulations with Unstructured Meshes, J. Comp. Phy., 84, pp. 174-206, (1989).

[5] DERVIEUX A., Steady Euler simulations using unstructured meshes, Von Karman Institute Lectures Series 85-04 (1985).

[6] DESIDERI J.A. - GOUDJO A. - SELMIN V. Third order numerical schemes for hyperbolic problems, rapport INRIA No 607 (1987)

[7] LANTERI S., Simulation d'écoulements aérodynamiques instationnaires sur une architecture S.I.M.D. massivement parallelle, Thèse (1991)

[8] DAUTRAY R. - LIONS J.L., Analyse mathématique et calcul numérique, Vol 1, pp. 68-127, Masson (1987)

[9] SHANKAR V. - HALL W.F. - MOHAMMADIAN A.H., A time-domain differential solver for electromagnetic scattering problems, Proceedings on the IEEE, Vol 77, pp. 709-721, No 5 (1989)

[10] JOLY P. - MERCIER B., Une nouvelle condition transparente d'ordre deux pour les équations de Maxwell en dimension trois, rapport INRIA No 1047 (1989).

[11] ENGQUIST B. - MAJDA A., Absorbing boundary conditions for acoustic and elastic waves calculation, Comm. Pure Appl. Math., Vol 32, pp. 313-357 (1979)

[12] PETITJEAN B. - LÖHNER R., Finite element solvers for Radar Cross-Section (RCS) calculations, AIAA paper 92-0455 (1992) 
[13] LÖHNER R. - AMBROSLANO J., A finite element solver for the Maxwell equations, paper presented at the GAMNI-SMAI Conference on Numerical Methods for the solution of Maxwell Equations, Paris (1989)

[14] BRISTEAU M.O. - GLOWINSKI R. - PERIAUX J., Scattering waves using exact controllability methods, AIAA paper 93-0460 (1993)

[15] YOUNG J.L. - BRUECKNER F.P., A time domain, weighted residual formulation of Maxwell's equations, AIAA paper 93-0462 (1993)

[16] BOWMAN J.J. - SENIOR T.B.A. - USLENGHI P.L.E., Electromagnetic and acoustic scattering by simple shapes, North Holland (1969)

[17] HARRINGTON R.F., Time-harmonic electromagnetic fields, Mc Graw-Hill (1961)

[18] LAX P.D. - PHILLIPS R.S., Scattering theory, Academic Press, New-York (1967)

[19] ABRAMOWITZ M. - STEGUN I., Handbook of mathemetical functions, Dover Publications (1968)

[20] NEDELEC J.C., Mixed finite elements in $\mathbb{R}^{3}$, Num. Math., Vol 35, pp.315-341 (1980)

[21] LEFLOUR G. et al., Calcul électromagnétique d'un avion complet par le code SPEC$T R E$, in proceedings of "Journées internationales de Nice sur les antennes", JINA, Nice (1990)

[22] HUH K.S. - SHU M. - AGARWAL R.K., A compact high-order finite volume timedomain/frequency-domain method for electromagnetic scattering, ALAA paper 92$0453(1992)$

[23] TAFLOVE A., Re-inventing electromagnetics: supcrcomputing solution of Maxwell's equations via direct time integration on space grids, AIAA paper 92-0333 (1992)

[24] FARHAT C. - FEZOUI L. - LANTERI S., Two-dimensionnal viscous Row computations on the Connection Machine : unstructured meshes, upwind schemes, and massively parallel computations, Comput. Meths. Appl. Mech. Engrg. (1992)

[25] DAHL E.D., Mapping and compiling communication on the connection machine system, Proc. Distr. Mem. Comp. Conf., Charleston (1990)

[26] Thinking Machine Corporation, Connection machine model CM-2: Technical summary, version $6.0(1990)$

[27] Thinking Machine Corporation, CM-Fortran optimisation notes: slicewise model, version $1.0(1991)$ 
Unité de Recherche INRIA Sophia Antipolis

2004, route des Lucioles - B.P. 93 - 06902 SOPHIA ANTIPOLIS Cedex (France)

Unité de Recherche INRIA Lorraine Technopôle de Nancy-Brabois - Campus Scientifique

615, rue du Jardin Botanique - B.P. 101 - 54602 VILLERS LES NANCY Cedex (France)

Unité de Recherche INRIA Rennes IRISA, Campus Universitaire de Beaulieu 35042 RENNES Cedex (France)

Unité de Recherche INRIA Rhône-Alpes 46, avenue Félix Viallet - 38031 GRENOBLE Cedex (France)

Unité de Recherche INRIA Rocquencourt Domaine de Voluceau - Rocquencourt - B.P. 105 - 78153 LE CHESNAY Cedex (France)

EDITEUR

INRIA - Domaine de Voluceau - Rocquencourt - B.P. 105 - 78153 LE CHESNAY Cedex (France)

ISSN $0249-6399$ 